\title{
InGaAs/InP double heterostructures on InP/Si templates fabricated by wafer bonding and hydrogen-induced exfoliation
}

\author{
A. Fontcuberta i Morral, ${ }^{\text {a) }}$ J. M. Zahler, and Harry A. Atwater \\ Thomas J. Watson Laboratory of Applied Physics, California Institute of Technology, \\ Pasadena, California 91125
}

\author{
S. P. Ahrenkiel and M. W. Wanlass \\ National Renewable Energy Laboratory, Golden, Colorado 80401
}

(Received 23 January 2003; accepted 3 November 2003)

\begin{abstract}
Hydrogen-induced exfoliation combined with wafer bonding has been used to transfer $\sim 600$-nm-thick films of (100) InP to Si substrates. Cross-section transmission electron microscopy (TEM) shows a transferred crystalline InP layer with no observable defects in the region near the bonded interface and an intimately bonded interface. InP and $\mathrm{Si}$ are covalently bonded as inferred by the fact that InP/Si pairs survived both TEM preparation and thermal cycles up to $620^{\circ} \mathrm{C}$ necessary for metalorganic chemical vapor deposition growth. The InP transferred layers were used as epitaxial templates for the growth of $\mathrm{InP} / \mathrm{In}_{0.53} \mathrm{Ga}_{0.47} \mathrm{As} / \mathrm{InP}$ double heterostructures. Photoluminescence measurements of the $\mathrm{In}_{0.53} \mathrm{Ga}_{0.47} \mathrm{As}$ layer show that it is optically active and under tensile strain, due to differences in the thermal expansion between InP and Si. These are promising results in terms of a future integration of Si electronics with optical devices based on InP-lattice-matched materials. (C) 2003 American Institute of Physics. [DOI: 10.1063/1.1637429]
\end{abstract}

Applications of InP-based materials are numerous, and thus integration of $\mathrm{InP}$ on Si may enable realization of powerful integrated III-V-on-Si systems. InP and its lattice matched quaternary counterpart $\mathrm{In}_{1-x} \mathrm{Ga}_{x} \mathrm{As}_{y} \mathrm{P}_{1-y}$ are direct gap semiconductors, which have high carrier mobilities, therefore finding applications in lasers, multijunction solar cells ${ }^{1}$ and high-speed devices. Additionally, they cover the low dispersion and minimum loss wavelengths for optical fiber communication at 1.3 and $1.5 \mu \mathrm{m}$, respectively, making them attractive materials for fabricating semiconductor lasers and detectors for telecommunications applications. However, InP is mechanically fragile, is not available in large substrates, and is expensive. Integrating InP thin films on $\mathrm{Si}$ substrates improves its mechanical strength and may also allow InP integration on large substrates by a process of tiling transferred thin films. Most importantly, a viable approach to InP/Si may enable cost-effective integration of infrared optoelectronic devices with well-established silicon electronics.

Because InP and Si have a large lattice mismatch (8.1\%), heteroepitaxial growth on $\mathrm{Si}$ has not yet been able to produce the high quality electronic material needed in optoelectronic devices, since the dislocation density is typically $10^{7} \mathrm{~cm}^{2} .^{2}$ In some cases densities of $10^{5} \mathrm{~cm}^{-2}$ can be reached by conformal growth, but this is only possible on very small areas. ${ }^{3}$ Other methods have been attempted to circumvent the restrictions of heteroepitaxial growth. However, most of these processes require an entire substrate to be consumed. ${ }^{4}$ Direct wafer bonding of III-V semiconductors to $\mathrm{Si}$ has been previously demonstrated. ${ }^{5,6}$ However, it would be more desir-

${ }^{a)}$ Present address: Laboratoire de Physique des Interfaces et des Couches Minces, UMR-7647, 91128 Palaiseau, France; electronic mail: annafm@poly.polytechnique.fr able to have a method for InP/Si integration in which the InP substrate can be repeatedly reused, rather than consuming one InP substrate per active InP-based device layer. Hydrogen-induced exfoliation and layer transfer has shown to be a successful method for Si film fabrication, which has motivated work on hydrogen-induced exfoliation of III-V semiconductors. ${ }^{7}$ By wafer bonding and hydrogen-induced layer transfer, a single InP substrate could be reused and implanted repeatedly allowing one InP bulk substrate to transfer many InP films, thus reducing the cost of InP/Si substrate fabrication. In this letter, we present results of the structural and epitaxial template properties of InP/Si heteroepitaxial templates fabricated by wafer bonding and hydrogen-induced layer transfer.

InP wafers were implanted at room temperature with 80 $\mathrm{keV} \mathrm{H}^{+}$to a dose of $2 \times 10^{17} \mathrm{~cm}^{-2}$. Following implantation, both the implanted $\mathrm{InP}$ and $\mathrm{Si}$ substrates were coated with photoresist to protect the bonding surfaces from scratches and particles. The substrates were then cleaved into $\sim 1-3 \mathrm{~cm}^{2}$ samples. The photoresist was removed and the samples were ultrasonically cleaned with acetone and methanol followed by de-ionized water to leave a smooth, particlefree surface. The surface was then rendered hydrophobic with $10 \% \mathrm{HF} / \mathrm{H}_{2} \mathrm{O}$ solution. Contact-mode, atomic-force microscopy (AFM) measurements showed that the surface roughness of both $\mathrm{InP}$ and $\mathrm{Si}$ is less than $0.5 \mathrm{~nm} \mathrm{rms}$, which enables intimate contact between the bonding surfaces. Bonding between the InP and Si samples is then initiated by contact at room temperature in an atmospheric ambient. The room temperature bonded samples were then annealed under nitrogen ambient to a temperature of $\sim 225^{\circ} \mathrm{C}$ under a uniaxial pressure of $\sim 0.5 \mathrm{MPa}$ to initiate hydrogen-induced layer exfoliation and layer transfer. By careful control of 


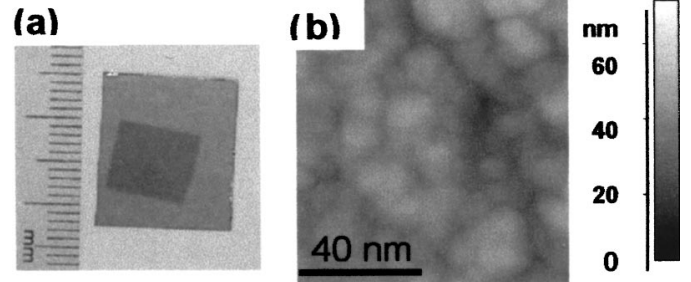

FIG. 1. (a) Optical micrograph of a $\sim 1 \mathrm{~cm}^{2}$ transferred InP layer on Si. (b) Contact mode atomic force microscopy image of the surface.

surface contamination and pressure uniformity, a complete layer transfer has been repeatedly demonstrated.

Figure 1(a) shows an optical image of a $\sim 1 \mathrm{~cm}^{2}$ InP transferred area. The as-transferred surface of the InP exhibits a $9-10 \mathrm{~nm}$ rms roughness, as measured by AFM and illustrated in Fig. 1(b). Micrographs of (110)-oriented cross sections obtained by cross-sectional transmission electron microscopy (TEM) are shown in Figs. 2(a)-2(c). A highresolution image of the interface in Fig. 2(b) indicates intimately bonded InP/Si interfaces. This, together with the ability of the sample to survive the TEM sample preparation process, suggests that InP and $\mathrm{Si}$ are covalently bonded. The image reveals the presence of a 4-nm-thick amorphous layer at the interface. Studies on wafer-bonded $\mathrm{InP} / \mathrm{Si}$ samples by Fourier-transform infrared spectroscopy suggest that water adsorbed at the bonded interface reacts upon annealing to $300{ }^{\circ} \mathrm{C}$ to form an interfacial layer of $\operatorname{InPO}_{x} \cdot{ }^{8}$ This behavior is consistent with previous work that shows that HF treated InP surfaces do not form a stable hydride passivation layer and are rapidly oxidized forming a surface to which water readily adsorbs. ${ }^{9}$ A dark-field cross-sectional (110) zone-axis TEM image [Fig. 2(a)] shows a 400-nm-thick, low-defect single crystal InP region next to the bonded interface. Figure 2(c) indicates, however, that a 200-nm-thick region nearest to the transferred InP surface appears dark in the bright-field image indicating a large defect density in this region, presumably due to damage from the implantation process.

The suitability of the as-cleaved InP transferred layer as a template for InP epitaxial growth was investigated by growing a lattice-matched $\mathrm{InP} / \mathrm{In}_{0.53} \mathrm{Ga}_{0.47} \mathrm{As} / \mathrm{InP}$ double heterostructure with atmospheric-pressure metalorganic vapor-

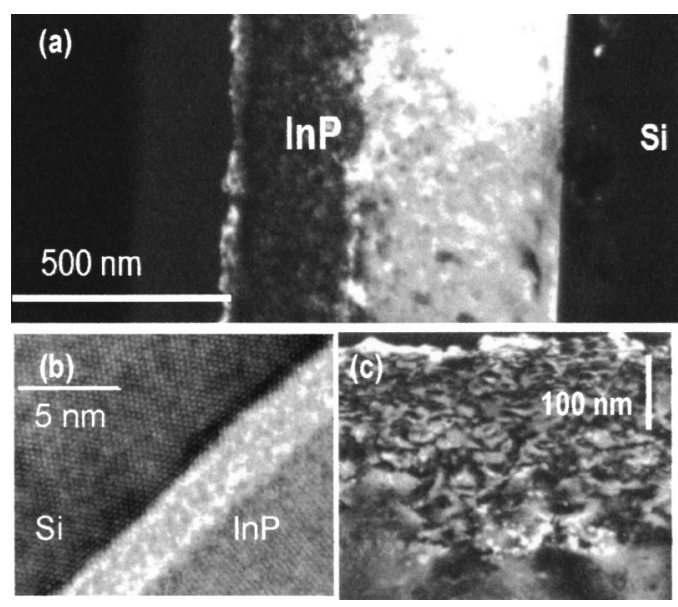

FIG. 2. (a) Dark-field image of a transferred layer of InP on Si. (b) Highresolution electron transmission micrograph of the interface of InP with Si.

(c) TEM micrograph of the damaged InP surface. InP thin films on (100) Si is possible by wafer bonding and
Downloaded $14 \mathrm{Dec} 2005$ to 131.215 .225 .171 . Redistribution subject to AlP license or copyright, see http://apl.aip.org/apl/copyright.jsp

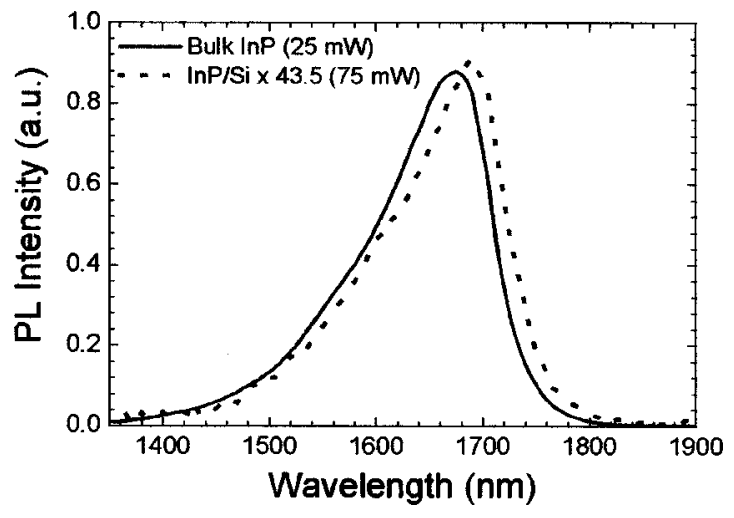

FIG. 3. $\quad$ InP/ $/ n_{0.53} \mathrm{Ga}_{0.47} \mathrm{As} / \mathrm{InP}$ double heterostructure photoluminescence spectra from a bulk InP control wafer and an InP/Si wafer bonded heterostructure.

phase epitaxy. The double heterostructure was grown simultaneously on an (100)InP/(100) Si structure and also on a bulk (100) InP control wafer at $620^{\circ} \mathrm{C}$. The V/III ratio was $\sim 120$ for the growth of InP and $\sim 10$ for $\operatorname{In}_{0.53} \mathrm{Ga}_{0.47}$ As. The growth rate was in both cases $100 \mathrm{~nm} / \mathrm{min}$. Each layer of the double heterostructure is $1000 \mathrm{~nm}$ thick. Photoluminescence spectra, shown in Fig. 3, were measured at room temperature with a $1064 \mathrm{~nm}$ pump wavelength with a $10 \mu \mathrm{m}$ diameter spot. A pump power of $25 \mathrm{~mW}$ was used on the control sample whereas a pump power of $75 \mathrm{~mW}$ was used for the double heterostructure grown on the transferred $\mathrm{InP} / \mathrm{Si}$. For purposes of comparing the line shape of the photoluminescence, the peak intensity of the spectrum from the waferbonded sample was normalized to that of the bulk InP control. The normalization factor was 43.5 , indicating a much weaker photoluminescence intensity for the $\mathrm{In}_{0.53} \mathrm{Ga}_{0.47} \mathrm{As}$ grown on the $\mathrm{InP}$ template. We attribute this to the fact that the surface of the transferred InP has a high density of dislocations resulting from residual ion implantation damage, as seen in Fig. 2(c). We anticipate that the defect density may be reduced by removal of the damaged layer. Importantly, the underlying InP layer appears to be defect-free, within the resolution of cross-sectional transmission electron microscopy, despite thermal cycling to $620^{\circ} \mathrm{C}$ of the InP/Si structure during epitaxial growth. Work on the improvement of the surface quality by chemical-mechanical polishing is in progress. The band edge photoluminescence peak has the same shape in both samples, but a slight bandgap shift of $\sim 15 \mathrm{~nm}$ to longer wavelengths is observed for the $\mathrm{In}_{0.53} \mathrm{Ga}_{0.47} \mathrm{As}$ grown on $\mathrm{InP} / \mathrm{Si}$, with respect to the control sample. This effect can be attributed to tensile strain in the grown films arising from the coefficient of thermal expansion mismatch of the InP and the underlying $\mathrm{Si}$ substrate. Indeed, as calculated by Kuo et al. ${ }^{10}$ such a shift would correspond to a tensile strain of $\sim 0.075 \%$. Based on the thermal expansion coefficients of InP and $\mathrm{Si}^{11}{ }^{11}$ the strain of an $\mathrm{InP}$ film on $\mathrm{Si}$ at room temperature as a function of the bonding temperature of $\mathrm{InP} / \mathrm{Si}$ pairs was calculated and is depicted in Fig. 4. This result suggests that the zero stress condition for the InP/Si interface is set by the onset of covalent bonding at $400{ }^{\circ} \mathrm{C}$ or higher, and thus the InP template is tensile strained about $\sim 0.07 \%$.

In conclusion, we have shown that integration of (100) InP thin films on (100) Si is possible by wafer bonding and 


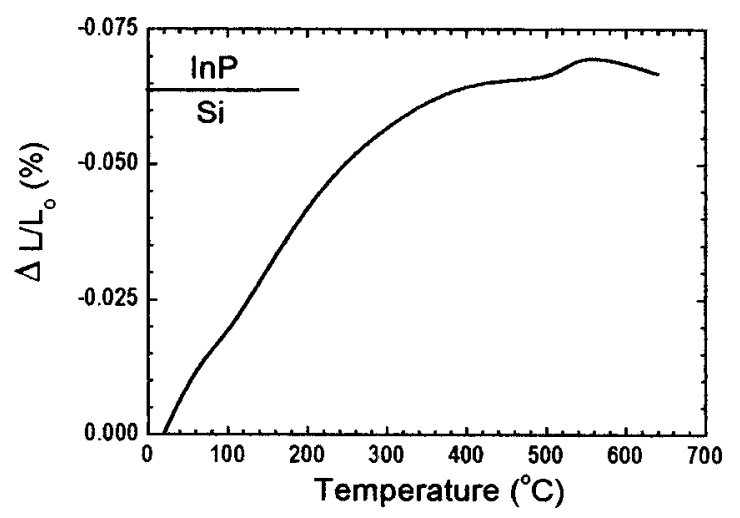

FIG. 4. Strain of a InP film on $\mathrm{Si}$ at room temperature as a function of bonding temperature.

hydrogen-induced exfoliation. The resulting InP layers are crystalline. Films survived TEM preparation and thermal cycles up to $620^{\circ} \mathrm{C}$ necessary for metalorganic chemical vapor deposition growth, indicating that the InP is covalently bonded to the Si substrate. Moreover, the films were used successfully as epitaxial templates for the growth of optically active $\mathrm{InP} / \mathrm{In}_{0.53} \mathrm{Ga}_{0.47} \mathrm{As} / \mathrm{InP}$ double heterostructures. These results show promise for the use of transferred InP layers as epitaxial templates for the growth of InP-based semiconductor heterostructures on $\mathrm{Si}$.
This work was supported by the National Renewable Energy Laboratory and NASA. The authors acknowledge L. Gedvilas for measuring the photoluminescence spectra and $\mathrm{J}$. Carapella for performing the metalorganic vapor phase epitaxy growths. Helpful discussion with C. G. Gahn, A. Risbud, and A. Ghaffari are gratefully acknowledged.

${ }^{1}$ P. R. Sharps, M. L. Timmons, J. S. Hills, and J. L. Gray, 26th IEEE PVSC, 1997.

${ }^{2}$ M. Sugo, Y. Takanashi, M. M. Aj-jassim, and M. Yamaguchi, J. Appl. Phys. 68, 540 (1990).

${ }^{3}$ O. Parillaud, E. Gil-Lafon, B. Gérard, P. Etienne, and D. Pribat, Appl. Phys. Lett. 68, 2654 (1996).

${ }^{4}$ D. L. Mathine, IEEE J. Sel. Top. Quantum Electron. 3, 952 (1997).

${ }^{5}$ T. Akatsu, A. Plöß1, R. Scholz, H. Stenzel, and U. Gösele, J. Appl. Phys. 90, 3856 (2001).

${ }^{6}$ D. Pasquariello and K. Hjort, IEEE J. Sel. Top. Quantum Electron. 8, 118 (2002).

${ }^{7}$ U. Goesele, M. Alexe, and Q. -Y. Tong, International Conference on Indium Phosphide and Related Materials Proceedings, 2000, p. 76.

${ }^{8}$ A. Fontcuberta i Morral, J. M. Zahler, Harry Atwater, M. M. Frank, Y. J. Chabal, S. P. Ahrenkiel, and M. Wanlass, Mater. Res. Soc. Symp. Proc. (to be published).

${ }^{9}$ O. Pluchery, J. Eng, Jr., R. L. Opila, and Y. J. Chabal, Surf. Sci. 502-503, 75 (2002).

${ }^{10}$ C. P. Kuo, S. K. Vong, R. M. Cohen, and G. B. Stringfellow, J. Appl. Phys. 57, 5428 (1985).

${ }^{11}$ Y. Touloukian, in Landolt-Bornstein Vol 17a, edited by O. Madelung, M. Schulz, and H. Weiss (IFI/Plenum, New York, 1970). 